\title{
Quantum Computing-Applications in Bioinformatics
}

\author{
Divya Baiskhiyar \\ Freelancer Researcher
}

\author{
Ravi Kumar \\ Enterprise Architect, Fisev
}

\begin{abstract}
Quantum computing is a promising field that emerged out of a combination of quantum physics and computer science. With ever expanding data across different areas, the conventional computer will run out of its capacity to handle such big data. Further, extracting the meaningful from big complex data still, accompany challenges with it. Quantum computing main goal is to provide such algorithms which are robust and faster in solving problems as compared to classical computers. In this paper, the limitations of a classical computer, basic features of quantum computing and its applications in the bioinformatics have been explored.
\end{abstract}

\section{General Terms}

Design, Complexity

\section{Keywords}

Quantum computing, Classical computing, Bioinformatics

\section{INTRODUCTION}

Computing is an indispensable part of all spheres of life and ranges from simple calculations to very complex problem issues. The continuous advancement in classical computing has been attributed to the powerful miniaturization of integrated circuits. However, the extent of such miniaturization and enhancing of chip capacity suffers from certain limitations. It has led to the search for alternatives and progress towards new type or next-generation computing such as quantum computing and molecular computing.

Quantum computing is an interdisciplinary research area that utilizes the principles of quantum mechanics. Quantum computer possesses unique abilities such as entanglement, superposition that enable to surpass some of the limitations of today's classical computer. For instance, their ability to perform extremely faster computations by reducing the number of calculations needed to complete a task. Further, it can help in solving problems that are currently unsolvable in varied disciplines such as bioinformatics, artificial intelligence, drug discovery, personalized medicine, biological system, and many others. The second section in this article describes a brief introduction of classical computing's limitations. The third section describes the basics properties of Quantum computing. Fourth section deals with potential applications of Quantum computing in the bioinformatics field. Finally, the last one includes concluding part of the paper.

\section{CLASSICAL COMPUTING AND ITS LIMITATIONS}

Classical computing involves operations on Boolean bits, i.e., $0 \mathrm{~s}$ and $1 \mathrm{~s}$. The current that flows through the conducting wires determine a particular state. For instance, when current is passing through then, it is said to be in logical 1(on) state or otherwise in logical 0 (off) state, these two forms a bit. Given a given ordered sequence of the bit as input results in an ordered sequence of bits as output. There has been significant growth in the power of classical computing that utilizes silicon-based integrated circuits. However, an increase in the computing power in such a way is not infinite and shown to follow a growth trend as explained by Moore's law. According to which the number of transistors is doubling in every 18 months with a corresponding decrease in transistors' size on the chip [1] since 1965. It attributes to significant limitations to using silicon-based technology which is as follows:

\subsection{Design complexity, density and speed limits}

All classical computing crams for achieving more and more speed via reducing the size of transistors and chips to minimizes the distance between the chips. As in turn it will lead to decrease in the time of transfer of instructions from one component to another during execution. The process demands to fit a higher number of transistors on a chip making it denser than before. Further, shrinking in chip size demands for more complex design to be made. But it has been predicted that in the near future the increase in transistors densities on shrinking size of chip will come to an end [2].

\subsection{Energy consumption and heat dissipation limit}

The proper functioning of transistors depends upon their size for switching between on and Off state. The switching speed depends upon their size due to the requirement of less power and resultant heat generation. The heat is generated while switching between these states. It means more switching will results in more heat at a specific time. Transistor's component (gate oxide) ability beyond a specific size (less than nanometer) to control the flow of electrons in the off state will be disturbed and results in the process of quantum tunneling [3]. Further, reduction in transistor's size in the range of nanometres and increased number of transistors will render the chip of no use in case any fabrication error occurs even at atom level. Additionally, a large amount of power dissipation will happen in the form of heat.

Thus, silicon-based classical computing is attaining its upper physical limits in terms of design complexity, speed, memory, energy consumption, density, and heat dissipation. Quantum computing is suggested as one of the alternatives to classical computing as it does not involve the use of transistors.

\section{QUANTUM COMPUTING AND ITS BASIC FEATURES}

Well-known high technology using companies like IBM, Microsoft, Google is investing in research and development towards quantum computing. Recently, IBM Q System OneTM has been developed by IBM which is the first commercial Quantum computer [4]. Following are some of the basic features possessed by quantum computing that make it lucrative: 


\subsection{Qubits}

In contrast to bit can have only one possible electronic state out of two $(|0\rangle$ and $|1\rangle)$ at a time in classical computing's, quantum computing leverages the advantage of subatomic particles where each state is represented by 'quantum bit' or 'qubit' that can attain 1,0 or any values in between them at a time. Such a system is called as complex two-state system where a state space has infinitely many possible states. Examples of the system include electron spin $-1 / 2$ or photon polarization or atom's ground state along with its excited state [5].

\subsection{Superposition}

In quantum computing, pure qubit $(|\Psi\rangle)$ can attain any superposition or linear combination of two basic states represented by Dirac notation $|0\rangle$ and $|1\rangle$, a $|0\rangle+b|1\rangle$ ), where $\mathrm{a}$ and $\mathrm{b}$ denote complex numbers given $|\mathrm{a}| 2+|\mathrm{b}| 2=1$. Further, two bits in classical computing can be indicated as 00, 01, 10 and 11. In contrast, a qubit can be represented by any of those numbers at the same time. As a result, greater number of qubits will lead to an exponential increase in the number of superposition that facilitates faster calculations involving very complicated numbers.

\subsection{Quantum parallelism}

The superposition feature imparts an ability to run a computation on possible classical states at a time that provides enormous computational power to quantum computing, and the process is referred to as quantum parallelism. Consequently, parallelism in quantum computing can perform some tasks shown to have advantages over classical computing [7]. Some of these advantages include factorization of large numbers and searching large databases.

\subsection{Entanglement}

The quantum entanglement refers to a process where a change in the state of one qubit is inseparable from the change in the state of others regardless of their spatial separation. Entanglement enables quantum computing to solve problems quickly to get on the right answer and fosters role in a variety of applications including teleportation, quantum cryptography, and others. For instance, quantum teleportation involves the transmission of stored quantum information based on the entangled quantum states adequately and correctly over reasonably long distances [8]

\section{APPLICATIONS OF QUANTUM COMPUTING IN THE BIOINFORMATICS}

In computer science, there are some problems that are being classified as NP-hard where a solution can only be got by trying out every possible solution. Traveling salesman problem is one such example where given a map with distances and routes for different cities need to be visited; the aim is to find out the shortest paths out of all. Likewise, several bioinformatics problems can fit into the category of NP-hard type. Some of them include Protein design problem [9], genome assembly [10], multiple sequence alignment with sum-of-pairs score (SP-score) [11],[12], motif discovery problems [13] discovery of disturbed pathways that lead to human diseases such as cancer. More detailed knowledge of these processes is critical to attaining a better understanding of the way dysregulation in some of the components that lead to diseases. Quantum computing with its power of superposition, entanglement, parallel processing represents a most-valued added tool to uncover the hidden information out of these processes at least to some level that currently cannot be achieved. Although, the applications of Quantum computing in bioinformatics is still in its nascent stage while some of the studies have been demonstrated its potential uses in solving a biological problem. For instance, quantum DWave Two $\mathrm{X}$ processor has been shown to map correctly selected transcriptors to correct binding sites on DNA [14]. Further, the use of D-Wave OneTM quantum computer has been used to determine the ground-state conformation of sixamino acid lattice protein models [15]. Another instance is where a system has been proposed that uses Shor Algorithm of Quantum computing with distinct Hierarchical Clustering Technique to obtain an approach for Cancer Disease Detection [16].

\section{CONCLUSION}

Quantum computing equipped with features such as superposition, entanglement, parallelism possesses the potential capability to tackle many problems that are classical computing unable to solve. Although quantum computing is yet in a nascent phase, it paves the way towards a new era of power computing which will enable to solve complicated problems including genome assembly, discovering proteinDNA binding interaction, disease detection and many more.

\section{REFERENCES}

[1] Moore, G, E. 1965. Cramming More Components onto Integrated Circuits. Electronics, 38(8), 114-117.

[2] Hruska, J. 2013. Intel's former chief architect: Moore's law will be dead within a decade.

[3] Seabaugh, A. 2013. The Tunneling Transistor. IEEE Spectrum. Retrieved from https://spectrum.ieee.org/semiconductors/devices/thetunneling-transistor

[4] IBM. 2019. IBM Unveils World's First Integrated Quantum Computing System for Commercial Use. Retrieved from https://newsroom.ibm.com/2019-01-08IBM-Unveils-Worlds-First-Integrated-QuantumComputing-System-for-Commercial-Use

[5] Bennett, H. C. 1995. Quantum information and computation. Physics Today. 24-30

[6] Dirac notation

[7] Sutor, B. 2018. Scientists Prove a Quantum Computing Advantage over Classical. IBM Research Blog.

[8] Olmschenk, S. et al. 2009. Quantum teleportation between distant matter qubits. Science, 323(5913), 486489

[9] Bazille, H. 2014. Protein design: a NP-hard problem in bioinformatics. Computer Science. 1-33

[10] Kapun, E., \& Tsarev, F. 2013. De Bruijn Superwalk with Multiplicities Problem is NP-hard. BMC bioinformatics, $14 \quad$ Suppl 5(Suppl 5), $\quad$ S7. doi:10.1186/1471-2105-14-S5-S7

[11] Just, W. 2001. Computational complexity of multiple sequence alignment with SP-score. Journal of Computational Biol biology 8(6), 615-623.

[12] Wang, L. and Jiang, T. 1994. On the complexity of multiple sequence alignment. Journal of Computational Biology, 1, 337-348. 
[13] Zaslavsky, E., \& Singh, M. 2006. A combinatorial optimization approach for diverse motif finding applications. Algorithms for molecular biology: AMB, 1, 13. doi:10.1186/1748-7188-1-13

[14] Li, R. Y., Felice, R. D., Rohs, R., \& Lidar, D. A. 2018. Quantum annealing versus classical machine learning applied to a simplified computational biology problem. Npj Quantum Information, 4(1), 14 https://doi.org/10.1038/s41534-018-0060-8
[15] Perdomo-Ortiz, A., Dickson, N., Drew-Brook, M., Rose, G., and Aspuru-Guzik, A. 2012. Finding low-energy conformations of lattice protein models by quantum annealing. Sci Rep, 2(571). doi: 10.1038/srep00571

[16] Jain, M and Chaturvedi, S.K. 2014. Quantum Computing Based Technique for Cancer Disease Detection System. J Comput Sci Syst Biol, 7, 095-102. doi: $10.4172 /$ jcsb. 1000143 